\title{
An improved model for calculating physical properties of confined fluid by considering adsorption film theory based on SWCF-VR equation of state
}

Fei Fang, Jinglian Gu, Xinqiang You*

Departement of Chemical Engineering, Laboratory of Chemical Process Intensification, College of Chemical Engineering, Fuzhou University, Fuzhou 350108, Fujian, China

* Corresponding author: E-mail: youxinqiang@gmail.com

\section{Support Information}

Table S1. The parameters of Langmuir model for alkanes.

\begin{tabular}{cccc}
\hline Adsorbate & Adsorbent & Temperature/K & b/Mpa ${ }^{-1}$ \\
\hline Methane & Zeolite-5A & 150 & 83.35 \\
Methane & Zeolite-5A & 160 & 65.46 \\
Methane & Zeolite-5A & 170 & 51.39 \\
Methane & Zeolite-5A & 180 & 40.33 \\
Ethane & ACF & 240 & 53.72 \\
Ethane & ACF & 260 & 30.90 \\
Ethane & ACF & 280 & 18.18 \\
Ethane & ACF & 300 & 11.09 \\
Propane & Silicalite & 280 & 693.9 \\
Propane & Silicalite & 300 & 492.2 \\
Propane & Silicalite & 320 & 345.5 \\
Propane & Silicalite & 340 & 238.6 \\
Butane & Silicalite & 360 & 712.3 \\
Butane & Silicalite & 380 & 355.1 \\
Butane & Silicalite & 400 & 233.8 \\
Butane & Silicalite & 420 & 192.7 \\
\hline
\end{tabular}


Table S2. The parameters of BET model for alkanes.

\begin{tabular}{cccc}
\hline Adsorbate & Adsorbent & Temperature/K & $\mathrm{C}$ \\
\hline Pentane & Silicalite & 360 & 1574 \\
Pentane & Silicalite & 380 & 1286 \\
Pentane & Silicalite & 400 & 996.8 \\
Pentane & Silicalite & 420 & 708.1 \\
Hexane & Silicalite & 420 & 1495 \\
Hexane & Silicalite & 440 & 1045 \\
Hexane & Silicalite & 460 & 595.2 \\
Hexane & Silicalite & 480 & 145.5 \\
Octane & Silicalite & 373 & 645.2 \\
Octane & Silicalite & 393 & 281.5 \\
\hline
\end{tabular}

Table S3. The parameters for the extended Antoine equation.*

\begin{tabular}{|c|c|c|c|c|c|c|c|c|c|}
\hline System & 1 & 2 & 3 & 4 & 5 & 6 & 7 & 8 & 9 \\
\hline Methane & 39.21 & -1324.4 & 0 & 0 & -3.44 & $3.10 \mathrm{E}-05$ & 2 & 90.69 & 190.56 \\
\hline Ethane & 51.86 & -2598.7 & 0 & 0 & -5.13 & $1.49 \mathrm{E}-05$ & 2 & 90.35 & 305.32 \\
\hline Propane & 59.08 & -3492.6 & 0 & 0 & -6.07 & $1.09 \mathrm{E}-05$ & 2 & 85.47 & 369.83 \\
\hline Butane & 66.34 & -4363.2 & 0 & 0 & -7.05 & $9.45 \mathrm{E}-06$ & 2 & 134.86 & 425.12 \\
\hline Pentane & 78.74 & -5420.3 & 0 & 0 & -8.83 & $9.62 \mathrm{E}-06$ & 2 & 143.42 & 469.70 \\
\hline Hexane & 104.65 & -6995.5 & 0 & 0 & -12.70 & $1.24 \mathrm{E}-05$ & 2 & 177.83 & 507.60 \\
\hline Octane & 96.08 & -7900.2 & 0 & 0 & -11.00 & $7.18 \mathrm{E}-06$ & 2 & 216.38 & 568.70 \\
\hline N-dodecane & 137.47 & -11976 & 0 & 0 & -16.70 & 8.09E-06 & 2 & 263.57 & 658 \\
\hline N-tetradecane & 140.47 & -13231 & 0 & 0 & -16.86 & $6.59 \mathrm{E}-06$ & 2 & 279.01 & 693 \\
\hline N-hexadecane & 156.06 & -15015 & 0 & 0 & -18.94 & $6.82 \mathrm{E}-06$ & 2 & 291.31 & 723 \\
\hline Eicosane & 203.66 & -19441 & 0 & 0 & -25.53 & $8.84 \mathrm{E}-06$ & 2 & 309.58 & 768 \\
\hline
\end{tabular}

$* \ln p_{i}^{*}, l=C_{1 i}+\frac{C_{2 i}}{T+C_{3 l}}+C_{4 i} T+C_{5 i} \ln T+C_{6 i} T^{C_{7 i}}$ for $C_{8 i} \leq T \leq C_{9 i}$. All data from Aspen Plus. 